\title{
The Age-Related Degeneration of Oligodendrocytes in the Hippocampus of the Senescence-Accelerated Mouse (SAM) P8: A Quantitative Immunohistochemical Study
}

\author{
Jun Tanake, ${ }^{a}$ Yasunobu Okuma, ${ }^{b}$ Koji Tomobe, ${ }^{b}$ and Yasuyuki NomurA ${ }^{*}, b$ \\ ${ }^{a}$ Northern Advancement Center for Science and Technology; Sapporo 060-0807, Japan: and ${ }^{b}$ Department of \\ Pharmacology, Graduate School of Pharmaceutical Sciences, Hokkaido University; Sapporo 060-0812, Japan. \\ Received October 14, 2004; accepted January 5, 2005
}

\begin{abstract}
The senescence-accelerated mouse (SAM) is known as a murine model for accelerated aging. The SAMP8 shows age-related deficits of learning and memory at an earlier age than control mice (SAMR1). We investigated the changes in oligodendrocytes in the brain of SAMP8, using immunohistochemistry for myelin basic protein (MBP) and 2',3'-cyclic nucleotide $3^{\prime}$-phosphodiesterase (CNP) as an oligodendrocyte marker. SAMP8 at 10 months old showed a decrease in MBP-immunoreactivity (IR) and CNP-IR in the hippocampal CA1 subfield, compared with SAMR1. There were no significant differences in MBP and CNP old in the cerebral cortex and the optic tract between SAMR1 and SAMP8 at 10 months. Furthermore, we measured the area of MBP-IR in the CA1 subfield of both strains and found that the area of MBP-IR in SAMP8 had decreased progressively with age, compared with SAMR1. These results suggest that age-related degeneration of oligodendrocytes had occurred in the hippocampus of SAMP8.
\end{abstract}

Key words senescence-accelerated mouse (SAM); myelin; hippocampus; oligodendrocyte; aging

The senescence-accelerated mouse (SAM) is a murine model of accelerated aging symptoms established by Takeda et $a l .{ }^{1)} \mathrm{SAM}$ is composed of eight aging-prone (SAMP) lines and three resistant (SAMR) lines. SAMP8 shows marked impairment of learning and memory. ${ }^{1-3)}$ We previously reported several neurochemical alterations in the hippocampus of aged SAMP8 such as i) the elevated amounts of glutamate and glutamine in the hippocampus ${ }^{4}{ }^{4}$ ii) the decreased release of acetylcholine and noradrenaline ${ }^{5,6)}$ in comparison to agematched controls from strain SAMR1. Yagi et al. ${ }^{7)}$ reported that the spongy degeneration in the brain stem of aged SAMP8 in histological analysis. They also reported that vacuolization in the brain stem was caused by changes to both oligodendrocytes and neural cell dendrites. Recently, it has been shown that expression of pro-inflammatory cytokines, such as interleukin 1- $\beta$ (IL1- $\beta$ ), interleukin-6 (IL-6) and tumor necrosis factor- $\alpha$ (TNF- $\alpha$ ) was elevated in the SAMP8 brain. ${ }^{8)}$ The oligodendrocytes are vulnerable to various factors that can easily cause cell death, including inflammatory cytokines, viruses and brain-blood barrier disruption. ${ }^{9)}$

An immunohistochemical study revealed that the activation of astrocytes and microglia occurred in the hippocampal CA1 subfield in addition to the brain stem of aged SAMP $8^{10,11)}$ however, the changes in oligodendrocytes in the hippocampus of aged SAMP8 are still unknown. Myelin basic protein (MBP) is a protein accounting for approximately $30 \%$ of the total protein of myelin, ${ }^{12,13)}$ and it is localized in the myelin and cell body of oligodendrocytes. ${ }^{4,15}$ ) $2^{\prime}, 3^{\prime}$-cyclic nucleotide $3^{\prime}$-phosphodiesterase (CNP) is an enzyme protein accounting for approximately $4 \%$ of the total myelin protein, and it is localized in the cell body and involved in oligodendrocyte processes, although its physiological role is not yet understood. ${ }^{16-18)}$ Melcangi et al. ${ }^{19)}$ reported that the MBP-IR in the sciatic nerve was markedly decreased in normal aging. In the present study, we examined the changes in oligodendrocytes in the brain of SAMP8, using MBP and CNP as an oligodendrocyte marker.

\section{MATERIALS AND METHODS}

Animals SAMP8 (SAMP8/Ta) and SAMR1 (SAMR1TA) were used for the immunohistochemical study; SAMR1 was used as the normal aging control. Both of the strains were bred under conventional conditions $\left(23 \pm 1{ }^{\circ} \mathrm{C}, 12 \mathrm{~h}\right.$ light/dark cycle with lights on at 7:00 a.m.) and given food (CE-2, Clea Japan, Inc., Tokyo, Japan) and tap water ad libitum. Three mice of both strains at each experimental age $(2,5$ and 10 months old) were used in this study. All animal experiments were carried out in accordance with the NIH Guide for the Care of Laboratory Animals and approved by the Animal Care and Use Committee at Hokkaido University.

SAMR1 and SAMP8 were anesthetized by sodium pentobarbital and were perfused transcardially with $4 \%$ paraformaldehyde in $0.1 \mathrm{~m}$ sodium phosphate buffer $(\mathrm{pH} 7.4$, $\mathrm{PB})$. The brains were removed and post-fixed in the same fixative for $2 \mathrm{~h}$.

Immunohistochemistry All immunohistochemical procedures were performed at room temperature. The fixed brains were embedded in paraffin wax blocks, and sections were $5 \mu \mathrm{m}$ thick. The sections were mounted on gelatincoated glass slides, deparaffined and washed with phosphatebuffered saline (PBS). All sections were incubated with $1 \%$ normal goat serum for $30 \mathrm{~min}$ for blocking. Then, some of the sections were incubated with rat anti-MBP antibody (diluted 1:5000, Chemicon, Temecula, U.S.A.) for $3 \mathrm{~d}$, while the other sections were incubated with mouse anti-CNP antibody (diluted $1: 1000$, Chemicon) overnight. The sections were washed with PBS and were incubated with biotinylated secondary antibody for $1 \mathrm{~h}$ and then with avidin-conjugated peroxidase for 30 min, using Vectastain $\mathrm{ABC}$ peroxidase kit (Vector Lab., Burlingame, CA, U.S.A.). The immunoreactions were visualized with 3,3-diaminobenzidine and counterstained with Mayer's hematoxylin. The photographs were taken using a Provis AX-80 microscope (Olympus, Tokyo, Japan). 
The Quantification of the Immunostained Area of MBP in the Hippocampus of SAMP8 and SAMR1 MBP immunostained area in the CA1 field of the hippocampus was quantified and expressed as $\mu \mathrm{m}^{2}$ using NIH image software version 1.61. The results were expressed as means \pm S.D.; statistical analysis was performed with a Student's $t$ test. Significant values were those with $p<0.05(*)$ and $p<0.01$ (**). Our measurements did not count the number of oligodendrocytes but only the area that was immunostained with MBP antibody in the hippocampal CA1 subfield.

\section{RESULTS}

Immunohistochemistry The MBP-IR was detected in the whole brain of SAMP8 and SAMR1 at all ages tested. In the absence of a primary antibody, the MBP-IR was diminished throughout (Fig. 1C). The coronal sections, that is, the areas of MBP-IR in the corpus callosum, of SAMP8 at 10 months old were thinner than that of SAMR1 (Fig. 1, arrow). However, the MBP-IR and morphology of myelin in the optic tract did not show any significant differences between the two strains (data not shown). We did not find any significant changes in the morphology of each MBP-IR fiber in the hippocampus or cerebral cortex of either strain. Although the MBP-IR was distributed in all layers in the hippocampus at all ages tested, the density of MBP-IR fibers was lower in aged SAMP8 (Fig. 1). Figure 2 shows the density of MBP-IR detected in the hippocampal CA1 subfield of SAMR1 and SAMP8. At 2 months old, the MBP-IR pattern was similar between the two strains (Figs. 2A, B). However, the MBP-IR of SAMP8 was decreased with age more progressively than that of SAMR1 (Figs. 2D, E).

Next, we investigated the immunohistochemical changes of CNP, an another oligodendrocyte marker. Like the case of MBP-IR, the densities of CNP-IR in the CA1 subfield of SAMP8 were lower than those in SAMR1 (Fig. 3).

The Quantification of the Immunostained Area of MBP in the Hippocampus of SAMP8 and SAMR1 We quantified the area of MBP-IR in the hippocampal CA1 subfield of
A

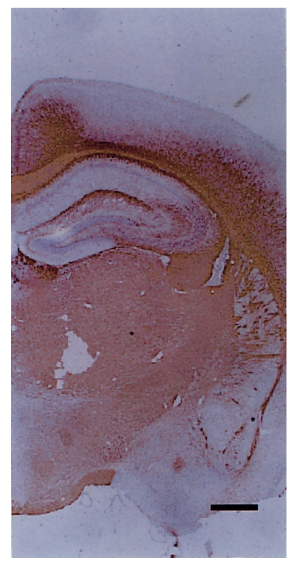

B

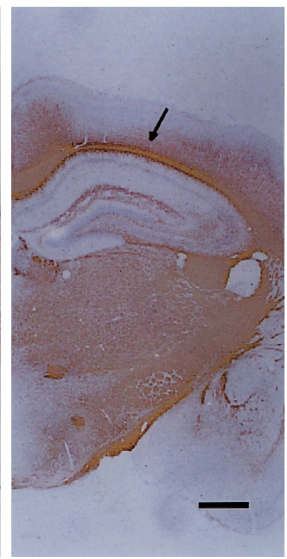

C

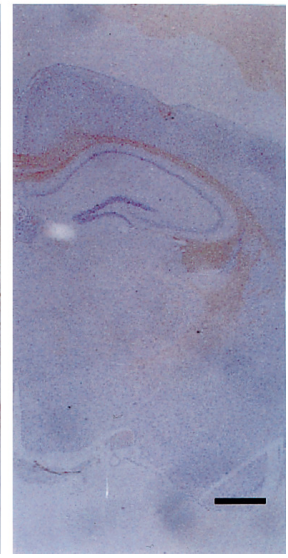

Fig. 1. The MBP-IR in the Coronal Sections of SAMR1 (A) and SAMP8 $(\mathrm{B}, \mathrm{C})$ Brain at 10 Months Old

The MBP-IR was detected intensely in white matter and scattered in gray matter in the brain of both strains. Note the thinned corpus callosum of the aged SAMP8 brain (arrow). The control section was demonstrated by omitting the primary antibody for MBP (C). Scale bar, $1 \mathrm{~mm}$.
SAMR1 and SAMP8 at all ages tested. Figure 4 shows the gravity score of the degeneration of oligodendrocytes in the hippocampal CA1 subfield of both strains at all ages tested. In the 2-month-old SAMP8, the degree of the MBP-IR area was $1954 \mu \mathrm{m}^{2}$ as scored by NIH image software, with a
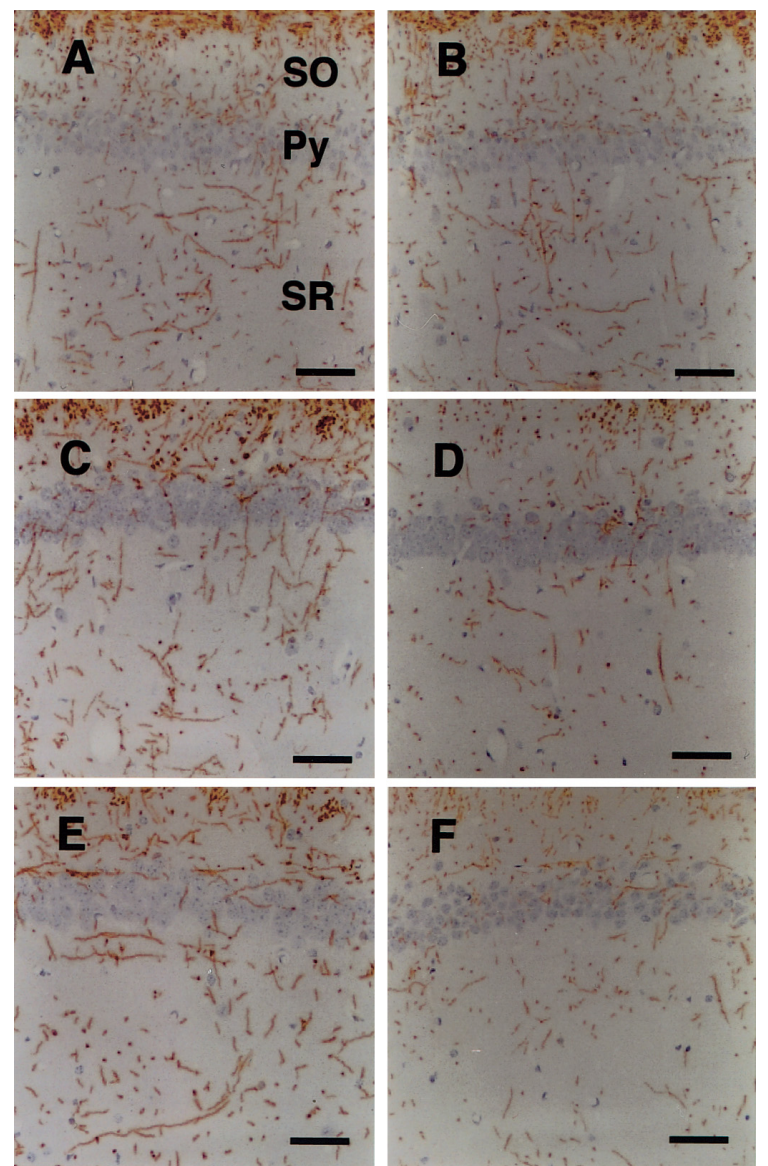

Fig. 2. The MBP Immunoreactivity in the Hippocampal CA1 Subfield of SAMR1 (A, C, E) and SAMP8 (B, D, E)

In 2-month-old SAMR1 (A) and SAMP8 (B), the MBP-IR was observed equally. However, the MBP-IR was decreased at 5 months old (D) and 10 months old (F) in SAMP8 compared with that of 5-month-old (C) and 10-month-old (E) SAMR1. Py, pyramidal cell layer; SO, stratum oriens; SR, stratum radiatum. Scale bars, $100 \mu \mathrm{m}$.
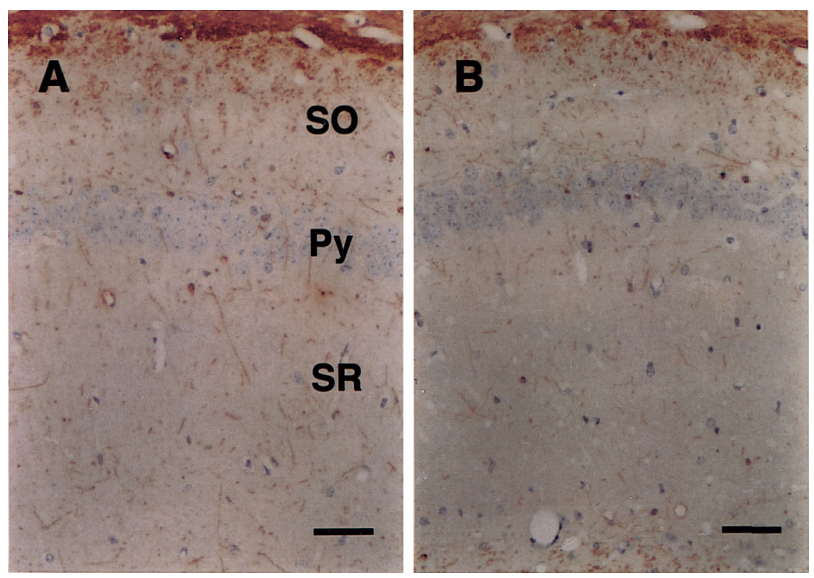

Fig. 3. The Immunoreactivity for CNP in the Hippocampal CA1 Subfield of SAMR1 (A) and SAMP8 (B) at 10 Months Old

The immunoreactivity for CNP was decreased the aged SAMP8 brain similar to the MBP-IR. Scale bar, $100 \mu \mathrm{m}$. 


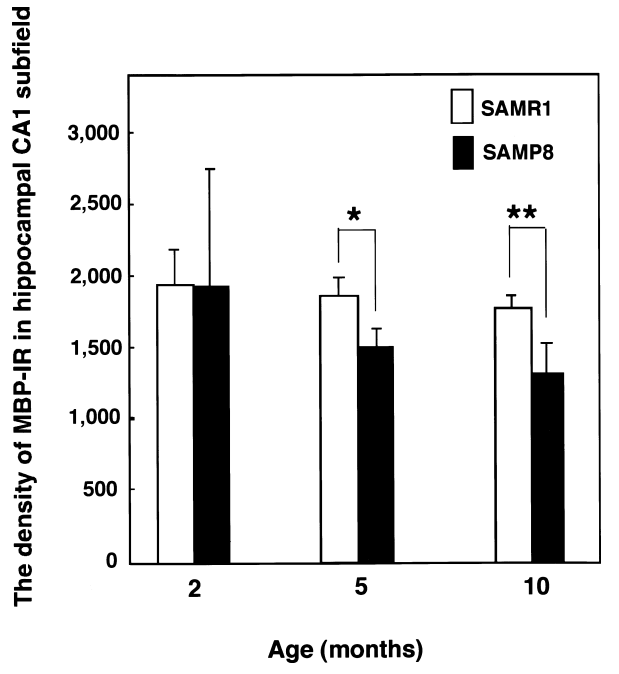

Fig. 4. The Comparison of MBP-IR in the Hippocampal CA1 Subfield of SAMR1 and SAMP8 at Each Age

MBP immunostained area in the CA1 field of the hippocampus was quantified and expressed as $\mu \mathrm{m}^{2}$ using NIH image software version 1.61. $* p<0.02, * * p<0.01$ (significant difference from the value of SAMR1, respectively).

range of scores from 993 to $2569 \mu \mathrm{m}^{2}$. There were no differences between SAMP8 and SAMR1. However the density of MBP-IR decreased gradually beginning at age 2 months, and the areas of MBP-IR of SAMP8 were decreased more progressively than those of SAMR1 with advancing age beginning at age 5 months $(p<0.02$ at 5 months old, $p<0.01$ at 10 months old).

\section{DISCUSSION}

In this study, we examined immunohistochemically for MBP in the brain of SAMP8 and SAMR1 mice at 2, 5 and 10 months old and tried to quantify the age-related changes of oligodendrocytes by measuring the area of MBP-IR in the hippocampus (CA1 subfield). The density of MBP- and CNP-IR nerve fibers in the CA1 subfield of aged SAMP8 mice at 10 months old was decreased greater than that of SAMR1 mice. In particular, the MBP-IR of SAMP8 was deescalated more progressively with age than that of SAMR1. SAMP8 mice show marked and age-related impairment of the memory and learning ability. The marked increase in astrocyte cells and microglia in the hippocampal CA1 subfield of aged SAMP8 has been shown by immunohistochemistry. ${ }^{11)}$ The MBP-IR in the CA1 subfield appeared to be in inverse proportion to the other glial cells with advancing age. In normal aged animals and in an experimental injured animal model, the changes in neuronal cells and glial cells in particular were investigated. For example, astrocytes proliferate and are activated with advancing age, showing astrogliosis. ${ }^{20,21)}$ Microglia and macrophages are also activated and proliferated, secreting various cytokines damaging to oligodendrocytes. ${ }^{22,23)}$

Yagi et al. ${ }^{7)}$ showed spongy degeneration in the brain stem of aged SAMP8 and also showed by electrical microscopy that the spongy degeneration of the brain stem was caused by myelin sheaths disruption. They also demonstrated that impairment of learning and memory was in proportion to the severity of spongy degeneration. ${ }^{24)}$
In the brain of aged normal animals, animals from an experimental injured animal model and aged SAMP8, astrocytes and microglia were activated, proliferated and secreted various inflammatory cytokines. IL1- $\beta$, TNF- $\alpha$ and IL-6 were expressed in aged SAMP8 brain and were increased compared with levels in SAMR1. ${ }^{8)}$ Since oligodendrocytes are injured by these cytokines directly, ${ }^{22)}$ the de-escalation of MBP-IR in the CA1 subfield of aged SAMP8 may suggest that oligodendrocytes are damaged by inflammatory cytokines.

In normal aging and in cases of diseases such as Alzheimer's disease and multiple sclerosis, oligodendrocytes and white matter are affected significantly. For example, it has been reported that the atrophy of white matter of the brain in normal aging, ${ }^{25,26)}$ decrease in the amount of myelin, ${ }^{27,28)}$ and marked decrease in the amount of MBP in the sciatic nerve were observed in aged rats. ${ }^{19)}$ With in vitro assay, the myelin sheaths of oligodendrocytes are degenerated with nitric oxide and inflammatory cytokines. ${ }^{22,29)}$ The level of CNP in the patient's brain in cases of Down syndrome and Alzheimer's disease was decreased. ${ }^{30)}$ However, it is not known whether SAMP8 is a model of these diseases and normal aging. The effects of normal aging on oligodendrocytes have been examined in detail using electron microscopy. The thickness of myelin sheaths was increased with advancing age, although the features of axons did not change. ${ }^{2631)}$ The myelin sheaths were broken, had separated from axons as they aged and had formed a balloon-like structure in the sciatic nerve. ${ }^{32)}$ Since we did not use electron microscopy for our analysis, we have not demonstrated whether the loss of MBP-IR was caused from the loss of oligodendrocytes or the loss of myelinated fibers including neuronal cells. In addition, the intracellular changes of oligodendrocytes of aged SAMP8 have not been investigated. However, MBP-IR in the optic nerve tract and the myelin in the optic nerve of aged SAMP8 were intact. These findings revealed the age-related degeneration of oligodendrocytes in the hippocampus of SAMP8 is probably caused by stress from outer elements, not from deficits of the oligodendrocytes themselves.

Acknowledgements We thank Dr. T. Iwanaga (Department of Anatomy, Graduate School of Veterinary Medicine, Hokkaido Univ., Sapporo, Japan) for technical advice. This study was supported by the Hokkaido collaboration of regional entities for the advancement of technological excellence, Japan Science and Technology Corporation. This research was also supported by Grants-in-Aid for Scientific Research from the Ministry of Education, Culture, Sports, Science, and Technology, Japan (Y.O., Y.N.).

\section{REFERENCES}

1) Takeda T., Hosokawa M., Higuchi K., J. Am. Geriatr. Soc., 39, 911919 (1981).

2) Miyamoto M., Kiyota Y., Yamazaki N., Nagaoka A., Matsuo T., Nagawa Y., Takeda T., Physiol. Behav., 38, 399-406 (1986).

3) Flood J. F., Morley J. E., Neurosci. Biobehav. Rev., 22, 1-20 (1998).

4) Kitamura Y., Zao X.-H., Ohnuki T., Takei M., Nomura Y., Neurosci. Lett., 137, 169-172 (1992).

5) Zao X.-H., Nomura Y., Int. J. Dev. Neurosci., 8, 267-272 (1990).

6) Zao X.-H., Nomura Y., Int. J. Dev. Neurosci., 10, 121-129 (1992).

7) Yagi H., Irino M., Matsushita T., Katoh S., Umezawa M., Tsuboyama 
T., Hosokawa M., Akiguchi I., Tokunaga R., Takeda T., J. Neuropath. Exp. Neurol., 48, 577-590 (1989).

8) Tha K. K., Okuma Y., Miyazaki H., Murayama T., Uehara T., Hatakeyama R., Hayashi Y., Nomura Y., Brain Res., 885, 25-31 (2000).

9) Espinosa de los Monteros, de Vellis, "The Role of Glia in Neurotoxicity." ed. by Aschner M., Kimelberg H. K., CRC Press, Boca Raton F.L., 1996, pp. 15- 46.

10) Kitamura Y., Yamanaka Y., Nagashima K., Nomura Y., "The SAM Model of Senescence," Excerpta Medica, Amsterdam, 1994, pp. $359-362$.

11) Kawamata T., Akiguchi I., Maeda K., Tanaka C., Higuchi K., Hosokawa M., Takeda T., Microsc. Res. Techniq., 43, 59-67 (1998).

12) Eng L. F., Chao F. C., Gerstl B., Pratt D., Tavaststjerna M. B., Biochemistry, 7, 4455-4465 (1968).

13) Trotter J. L., Lieberman L., Margolis F. L., Agrawal H. C., J. Neurochem., 36, 1256-1262 (1981).

14) Hartman B. K., Agrawal H. G., Kalmbach S., Shearer W. T., J. Comp. Neurol., 188, 273-290 (1979).

15) Hartman B. K., Agrawall H. C., Agrawall D., Kalmbach S., Proc. Natl. Acad. Sci. U.S.A., 79, 4217-4220 (1982).

16) Sheedlo H. J., Spinkle T. J., J. Neurol. Sci., 67, 35-44 (1985).

17) Vogel U. S., Thompson R. J., J. Neurochem. 50, 1667-1677 (1988).

18) Reinikainen K. J., Pitkanen A., Riekkinen P. J., Neurosci. Lett., 106, 229-232 (1989).

19) Melcangi R. C., Magnaghl V., Cavarreta I., Riva M. A., Piva F., Mar- tini L., Exp. Gerontol., 33, 827-836 (1998).

20) Bronson R. T., Lipman R. D., Harrison D. E., Brain Res., 609, 124 128 (1993).

21) Amenta F., Bronzetti E., Sabatini M., Vega J. A., Microscop. Res. Tech., 43, 29-33 (1998).

22) Hartung H. P., Jung S. F., Stoll G., Zielasek J., Schmidt B., Archelos J. J., Toyka K. V., J. Neuroimmunol., 40, 197-210 (1992).

23) Felzien L. K., McDonald J. T., Gleason S. M., Berman N. E. J., Kein R. M., Brain Res., 890, 137-146 (2001).

24) Yagi H., Akiguchi I., Ohta A., Yagi N., Hosokawa M., Takeda T., Brain Res., 791, 90-98 (1998).

25) Guttmann C. R., Jolesz F. A., Kikinis R., Kilianny R. J., Moss M. B., Sandor T., Albert M. S., Neurology, 50, 972—978 (1998).

26) Peters A., J. Comp. Neurol., 371, 153-163 (1996).

27) Lintl P., Braak H., Acta Neuropatol., 61, 178-182 (1983).

28) Kemper T. L., "Clinical Neurology and Aging" ed. by Albert M. L., Knoefel J. E., Oxford Uiversity Press, Oxford, 1994, pp. 3-67.

29) Mitrovic B., Ignarro L. J., Montestruque S., Smoll A., Merril J. E., Neuroscience 61, 575-585 (1994).

30) Vlkolinsky R., Cairns N., Fountoulakis M., Lubec G., Neurobiol. Aging, 22, 547-553 (2001).

31) Peters A., Moss M. B., Sethares C., J. Comp. Neurol., 419, 364-376 (2000).

32) Knox C. A., Kokmen E., Dyck P. J., J. Neuropathol. Exp. Neurol., 48, 119-139 (1989). 